BASIC RESEARCH

\title{
Heterozygous disruption of SERCA2a is not associated with impairment of cardiac performance in humans: implications for SERCA2a as a therapeutic target in heart failure
}

\author{
B M Mayosi, A Kardos, C H Davies, F Gumedze, A Hovnanian, S Burge, H Watkins
}

Heart 2006;92:105-109. doi: 10.1136/hrt.2004.051037

See end of article for authors' affiliations

Correspondence to: Professor Hugh Watkins, Department of

Cardiovascular Medicine, John Radcliffe Hospital, Oxford OX3 9DU, UK: hugh.watkins@cardiov.ox. ac.uk

Accepted 30 March 2005 Published Online First 21 April 2005
Objective: To verify whether a deficiency in the cardiac sarcoplasmic reticulum pump SERCA2a causes cardiac dysfunction in humans.

Design: Cardiac performance was measured in a serendipitous human model of primary SERCA2a deficiency, Darier's disease, an autosomal dominant skin disorder caused by mutations inactivating one copy of the ATP2A2 gene, which encodes SERCA2a.

Methods: Systolic and diastolic function and contractility were assessed by echocardiography at rest and during exercise in patients with Darier's disease with known mutations. Fourteen patients with Darier's disease were compared with 14 normal controls and six patients with dilated cardiomyopathy with stable heart failure.

Results: Resting systolic and diastolic function was normal in patients with Darier's disease and in controls. The increase in systolic function during exercise was not different between patients with Darier's disease and normal controls; neither was there a difference in contractility. As expected, patients with dilated cardiomyopathy had impaired diastolic and systolic function with depressed contractility at rest and during exercise.

Conclusion: Contrary to expectations, heterozygous disruption of SERCA2a is not associated with the impairment of cardiac performance in humans. Attempts to increase SERCA2a levels in heart failure, although showing promise in rodent studies, may not be addressing a critical causal pathway in humans.
$\mathrm{T}$ he sarco(endo)plasmic reticulum $\mathrm{Ca}^{2+}$ ATPase 2 (ATP2A2) gene encodes, by alternative splicing of mRNA, both the cardiac sarcoplasmic reticulum pump (SERCA2a) and the SERCA2b calcium pump, which is expressed in all tissues. ${ }^{1}$ In the heart, the sarcoplasmic reticulum provides the bulk of the $\mathrm{Ca}^{2+}$ release responsible for initiating cardiac contraction and subsequently reuptake of $\mathrm{Ca}^{2+}$ by the SERCA2a pump produces active myofilament relaxation. ${ }^{2}$ Substantial evidence indicates that SERCA2a protein level and function are reduced by about $30-50 \%$ in the failing human heart, in parallel to both the depressed contraction and impaired relaxation. ${ }^{3-7}$ One of the characteristic features of sarcoplasmic reticular function is that its contribution to cardiac performance becomes more prominent at higher heart rates. ${ }^{8}$ A parallel is observed in heart failure, where cardiac function is often normal at rest but fails to increase with exercise..$^{4}$ Given this, the reduction in SERCA2a activity is certainly plausible as a primary causal abnormality in heart failure ${ }^{5-79}$ but, alternatively, the reduction may be a compensation or an epiphenomenon. ${ }^{10}$

Support for a causal role of reduction in SERCA2a in heart failure has been provided by studies in mouse knockout models, as impairment of cardiac $\mathrm{dP} / \mathrm{dT}_{\max }$ (a measure of contractile function) is seen in mice with a single copy of the gene inactivated. ${ }^{11}{ }^{12}$ Accordingly, therapeutic upregulation of SERCA2a protein level is being explored. ${ }^{13}{ }^{14}$ However, the effects of primary reductions in SERCA2a on cardiac performance in humans remain unknown. Of note, attempts to identify pathogenic mutations in SERCA2a in human heart failure have been unsuccessful. ${ }^{15}$ We have recently identified a serendipitous human knockout model of SERCA2a, Darier's disease, a rare autosomal dominant skin disorder with no known cardiac associations that is caused by heterozygous mutation of the ATPA2A gene. ${ }^{16}{ }^{17}$ In Darier's disease, the majority of mutations occur in the region of ATP2A2 that encodes both the SERCA2a cardiac isoform and the ubiquitous SERCA2b isoform, which is important in the skin; these mutations result in complete or partial loss of function of the mutated calcium pumps. ${ }^{11} 19$ Thus, Darier's disease provides a unique opportunity to study the effects of primary SERCA2a deficiency on cardiac performance in humans.

\section{METHODS}

We investigated whether heterozygous disruption of SERCA2a in patients with Darier's disease results in clinically measurable effects in cardiac performance, especially at higher heart rates during exercise. The Central Oxford Research Ethics Committee approved the study, and all participants gave written informed consent. Fourteen patients with Darier's disease were compared with 14 normal controls. The normal controls were recruited from members of staff at the John Radcliffe Hospital and were matched for age of the patients with Darier's disease. To reduce the incidence of coexisting cardiovascular disease, only subjects $<60$ years with no history of cardiac disease or hypertension were studied. Patients with coronary artery disease were excluded. Echocardiography was performed in the resting state and systolic and diastolic performance was measured. The reproducibility estimates for echocardiographic measurements in our laboratory have been reported previously. ${ }^{20}$

Abbreviations: DT, deceleration time of the $E$ wave; FS, fractional shortening; $\mathrm{VO}_{2}$, oxygen consumption 
Systolic function was measured by $M$ mode fractional shortening (FS). The ratio of the Doppler transmitral early (E) to atrial (A) filling velocities (E:A ratio) and the deceleration time of the E wave (DT) were used to measure diastolic function.

Subjects performed a symptom limited supine bicycle exercise test with repeat echocardiography at four minute intervals to assess changes in cardiac performance during exercise. $^{21}$ Echocardiographic imaging during the supine bicycle exercise test was accomplished with a specialised bed with an integrated bicycle ergometer that provided lateral bed rotation for optimisation of echocardiographic windows (SBSS 1000, Redbud Medical Systems, Cashmere, Washington, USA). A single operator (AK) performed echocardiography and one reader (BMM) scored the images; the reader was blinded to the allocation group of the participants at the time of reading.

The protocol called for a workload of $50 \mathrm{~W}$ in the first stage of exercise and $25 \mathrm{~W}$ increments in subsequent stages. Heart rate and blood pressure were measured during the fourth minute of every stage of exercise. Oxygen consumption $\left(\mathrm{VO}_{2}\right)$ was measured by standard equipment that incorporated a computer for the calculation of $\mathrm{VO}_{2}$ every 30 seconds during exercise. FS and the FS-end systolic wall stress relation were used as non-invasive measures of systolic function response and contractility during exercise, respectively. ${ }^{22}{ }^{23}$ End systolic wall stress of the left ventricle was calculated by the formula $\sigma_{\mathrm{ES}}=[(\mathrm{ESP}) \times(\mathrm{ESD}) \times(1.35)] /[(4) \times(\mathrm{EST}) \times(1+\mathrm{EST} /$ ESD) $]$, where $\sigma_{\mathrm{ES}}$ is end systolic wall stress in $\mathrm{g} / \mathrm{cm}^{2}$; ESP is end systolic blood pressure in mm Hg; ESD and EST are end systolic diameter and wall thickness, respectively, in $\mathrm{cm}$; and 1.35 is the factor to convert pressure from $\mathrm{mm} \mathrm{Hg}$ to $\mathrm{g} / \mathrm{cm}^{2}$. End systolic pressure was calculated as $[2 \times$ systolic blood pressure + diastolic blood pressure]/3. ${ }^{23}$ The cardiac performance of patients with Darier's disease at rest and during exercise was also compared with that of six patients with stable heart failure caused by idiopathic dilated cardiomyopathy (New York Heart Association class II-III). The patients with heart failure acted as positive controls to determine whether the non-invasive methods used in the study were sufficient to detect an impaired cardiac performance response to exercise.

Data were statistically analysed with the Stata package (StataCorp LP, College Station, Texas, USA). The differences in means of physiological characteristics of the patient groups were compared by the unpaired Student's $t$ test. A repeated measures analysis of variance was used to compare the cardiac response to exercise between groups. FS was determined at rest, in at least two stages during exercise, and at one minute after cessation of exercise, such that all participants had at least four sequential measurements

Table 1 ATP2A2 mutations in patients with Darier's disease ${ }^{17,24}$

\begin{tabular}{|c|c|c|c|c|}
\hline Patient & Mutation & Exon & Consequence & Protein domain \\
\hline 1 & E412G & 10 & Missense & ATP binding \\
\hline 2 & 2134delA & 15 & PTC $(+43 a a)$ & Phosphorylation \\
\hline 3 & W551X & 13 & PTC & ATP binding \\
\hline 4 & 2102del36 & 15 & In-frame deletion & Phosphorylation \\
\hline 5 & T357K & 8 & Missense & Phosphorylation \\
\hline 6 & S920Y & 19 & Missense & M8-M9 loop \\
\hline 8 & D702N & 15 & Missense & Phosphorylation \\
\hline 11 & 2608delAG & 18 & PTC (+4aa) & M7-M8 loop \\
\hline $13^{*}$ & 2026insG & 14 & PTC (+3aa) & Phosphorylation \\
\hline $14^{*}$ & 2026 ins $G$ & 14 & PTC (+3aa) & Phosphorylation \\
\hline
\end{tabular}

*Patients 13 and 14 are related.

$M$, transmembrane domain; PTC $+n$, premature termination codon at $n$ amino acid downstream of the mutation.
Table 2 Physiological characteristics of patients with Darier's disease and normal controls

\begin{tabular}{llll}
\hline & $\begin{array}{l}\text { Darier group } \\
(\mathbf{n}=14)\end{array}$ & $\begin{array}{l}\text { Control group } \\
(\mathbf{n}=14)\end{array}$ & p Value \\
\hline Age (years) & $46.9(2.4)$ & $46.2(3.3)$ & 0.849 \\
LVMI (g/m ${ }^{2}$ ) & $116.9(5.9)$ & $113.5(7.7)$ & 0.728 \\
Resting FS (\%) & $39.7(1.6)$ & $42.2(2.3)$ & 0.770 \\
Resting E:A ratio & $1.4(0.1)$ & $1.3(0.07)$ & 0.747 \\
Resting DT (ms) & $210.2(12.9)$ & $216.2(12.9)$ & 0.369 \\
Exercise duration (min) & $11.4(1.0)$ & $15.0(1.0)$ & $0.020^{*}$ \\
$\mathrm{HR}_{\text {max }}$ (beats/min) & $128.9(3.5)$ & $144.8(5.7)$ & $0.023^{*}$ \\
$\mathrm{Vo}_{\text {2peak }}(\mathrm{ml} / \mathrm{kg} / \mathrm{min})$ & $17.7(1.0)$ & $23.7(1.9)$ & $0.007^{*}$ \\
\hline
\end{tabular}

Values are means (SEM).

Echocardiographic data were available for 13 of the control subjects. *Significant differences.

$D T$, deceleration time of the $E$ wave; $E: A$, ratio of early $(E)$ to atrial $(A)$ transmitral Doppler velocity; FS, fractional shortening; $H R_{\max }$, maximum heart rate; LVMI, left ventricular mass index (left ventricular mass/body surface area); $\mathrm{VO}_{2 \text { peak, }}$ peak oxygen consumption.

available for analysis. The data were arranged with four variables so that four FS measurements were recorded for each participant. FS was defined as a within-subject factor and study group was defined as a between-subject factor. The hypothesis regarding the between-subject factor was tested.

Left ventricular contractile performance was assessed by the FS end systolic wall stress relation by plotting FS as a function of end systolic wall stress at baseline, during exercise, and at one minute after cessation of exercise. In the comparison of contractile performance between the groups, an analysis of covariance was used to determine whether the slopes of the regression lines obtained from the groups were significantly different. A probability value of $\mathrm{p}<0.05$ was considered significant.

\section{RESULTS}

Mean (SD) age was 46.9 (9.0) years in the Darier's group and 46.2 (11.8) years in the normal controls. There were seven men and seven women in the Darier's group, and 10 men and four women in the normal control group. The causative ATP2A2 mutations had been identified in 10 of 14 patients with Darier's disease; the mutations predict premature termination of translation in five patients, non-conservative missense mutations in functional domains in four, and an inframe deletion in one (table 1). ${ }^{17}$ The mutations disrupt important domains of the SERCA2 molecule and result in complete or partial loss of function of the mutated calcium pumps. ${ }^{18} 19$

Echocardiographic data was of adequate quality from 13 of the 14 normal controls and from all 14 of the patients with Darier's disease. The patients with Darier's disease had normal resting cardiac function as shown by the indices of diastolic (DT and E:A ratio) and systolic function (FS) that

Table 3 Selected physiological characteristics of patients with Darier's disease and normal female controls.

\begin{tabular}{llll}
\hline & $\begin{array}{l}\text { Darier group } \\
(\mathbf{n = 7})\end{array}$ & $\begin{array}{l}\text { Control group } \\
(\mathbf{n}=\mathbf{4})\end{array}$ & $\mathbf{p}$ Value \\
\hline Age (years) & $46.1(4.5)$ & $42.8(6.8)$ & 0.675 \\
LVMI (g/m ${ }^{2}$ ) & $107.4(8.1)$ & $93.5(9.8)$ & $0.006^{*}$ \\
Resting FS (\%) & $38.6(1.7)$ & $46.0(2.0)$ & $0.026^{*}$ \\
Resting E:A ratio & $1.5(0.2)$ & $1.3(0.3)$ & 0.312 \\
Resting DT (ms) & $204.3(14.5)$ & $225.8(15.7)$ & 0.528 \\
Exercise duration (min) & $9.1(0.6)$ & $10.1(0.7)$ & 0.368 \\
$\mathrm{HR}_{\text {max }}$ (beats/min) & $135.4(5.5)$ & $164.0(4.3)$ & $0.025^{*}$ \\
$\mathrm{VO}_{\text {2peak }}(\mathrm{ml} / \mathrm{kg} / \mathrm{min})$ & $16.7(1.2)$ & $25.5(5.1)$ & $0.057^{*}$ \\
\hline
\end{tabular}

Values are means (SEM).

*Significant differences. 


\begin{tabular}{|c|c|c|c|}
\hline & $\begin{array}{l}\text { Darier group } \\
(n=7)\end{array}$ & $\begin{array}{l}\text { Control group } \\
(n=10)\end{array}$ & p Value \\
\hline Age (years) & $47.7(2.2)$ & $47.7(2.3)$ & 0.992 \\
\hline $\operatorname{LVMI}\left(\mathrm{g} / \mathrm{m}^{2}\right)$ & $126.3(7.3)$ & $122.3(9.1)$ & 0.751 \\
\hline Resting FS (\%) & $40.7(2.7)$ & $40.4(3.1)$ & 0.302 \\
\hline Resting $\mathrm{E}: \mathrm{A}$ ratio & $1.2(0.09)$ & $1.3(0.09)$ & 0.882 \\
\hline Resting DT (ms) & $216.1(22.3)$ & $211.9(17.6)$ & 0.946 \\
\hline Exercise duration (min) & $13.6(1.5)$ & $15.2(1.0)$ & 0.161 \\
\hline $\mathrm{HR}_{\max }$ (beats/min) & $122.3(2.7)$ & $136.2(6.2)$ & 0.083 \\
\hline $\mathrm{VO}_{2 \text { peak }}(\mathrm{ml} / \mathrm{kg} / \mathrm{min})$ & $18.7(1.6)$ & $21.1(1.3)$ & 0.098 \\
\hline
\end{tabular}

were similar to those of the normal control group (table 2). As a group the patients with Darier's disease were significantly undertrained compared with the normal controls as shown by their tendency to tire early (significantly shorter duration of exercise, $\mathrm{p}=0.020)$ and the achievement of a significantly lower peak $\mathrm{VO}_{2}(\mathrm{p}=0.007)$ (table 2$)$. These significant differences in baseline physiological characteristics between the patients with Darier's disease and normal controls were due to inadequate matching of the female members of the groups for heart size, resting FS, peak heart rate, and peak $\mathrm{VO}_{2}$ (table 3). Therefore, men from each group who were well matched with regard to baseline physiological characteristics and exercise performance (table 4) were compared.

A repeated measures analysis of variance showed no differences in FS between patients with Darier's disease and normal controls during exercise $(p=0.689$ ) (fig 1 ). However, the increase in FS over the stages of exercise was significant in both groups $(p<0.0001)$. There was no interaction between male study group and stage of exercise, suggesting that the increase in systolic function was of similar magnitude $(p=0.308)$. FS was significantly related to end systolic stress among patients with Darier's disease and normal controls $(\mathrm{p}<0.0001)$ (fig 2$)$. However, there was no interaction between study group and end systolic stress $(p>0.05)$, indicating that cardiac contractility during exercise did not differ significantly between the two groups.

As the participants in this study had no cardiac symptoms we used a non-invasive method to measure contractility, which may potentially have lacked sensitivity for the

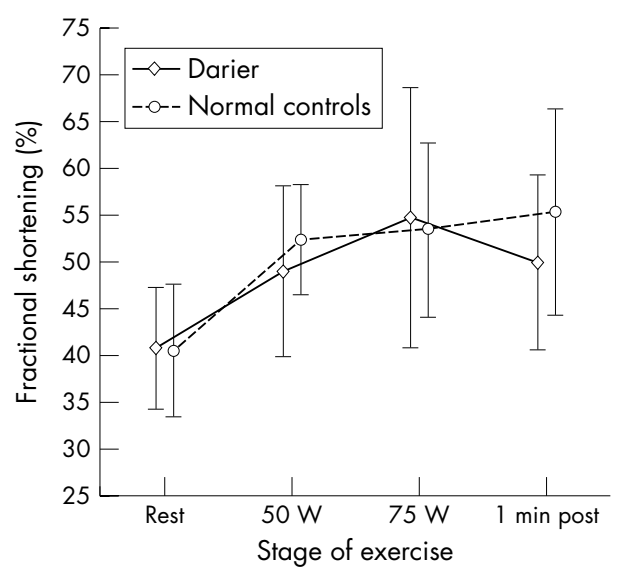

Figure 1 Left ventricular systolic function at different stages of exercise in men with Darier's disease and normal male controls was determined by echocardiography at rest and in response to exercise with increasing workloads $(50 \mathrm{~W}, 75 \mathrm{~W})$ and one minute after cessation of exercise. Mean (SEM).

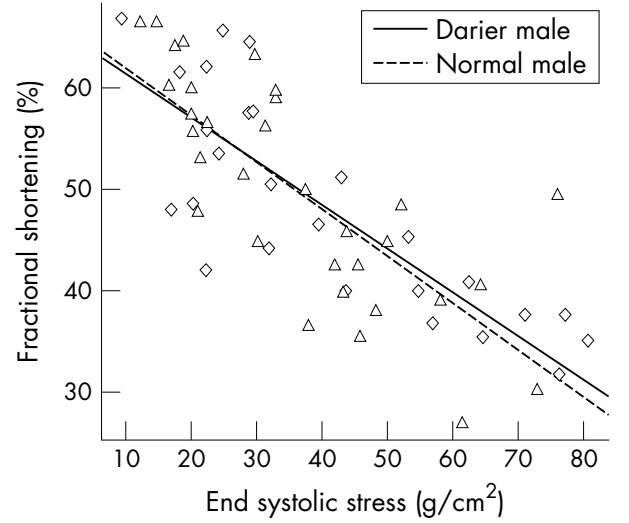

Figure 2 Contractility in men with Darier's disease and normal male control measured by the slope of the systolic shortening-end systolic stress relation. $\diamond$, Data points for male patients with Darier's disease; $\Delta$, data points for normal male controls.

detection of abnormalities in cardiac performance. Therefore, we studied cardiac performance in patients with heart failure who acted as positive controls. Four women and two men with stable mild to moderate heart failure (New York Heart Association class II-III) caused by idiopathic dilated cardiomyopathy with a mean (SD) age of 62.0 (9.2) years were studied. Resting systolic and diastolic function was, as expected, significantly impaired in the patients with heart failure (FS 22.7 (9.4)\%; E:A 1.03 (0.45); and DT 161.5 (36.2)). A repeated measures analysis of variance showed a significant difference in the FS in different stages of exercise between patients with Darier's disease and those with heart failure $(\mathrm{p}=0.0002)$ (fig 3). FS also increased significantly over the stages of exercise in both groups $(p<0.0001)$. However, there was a significant interaction between study group and stage of exercise $(\mathrm{p}=0.01)$-that is, the patients with Darier's disease had a different FS response pattern to exercise from that of the patients with heart failure. FS was found to be significantly related to end systolic stress among patients with Darier's disease and those with heart failure $(\mathrm{p}<0.0001$ ) (fig 4). However, there was also a significant interaction between study group and the FS-end systolic stress relation $(p=0.01)$, confirming that patients with heart failure had impaired cardiac contractility compared with the patients with Darier's disease. This confirms that the methods used in our study would be able to detect clinically relevant impairment in cardiac performance in patients with Darier's disease, if it were present.

\section{DISCUSSION}

Given the postulated role of SERCA2 in heart failure, it is surprising that no overt cardiac phenotype is present in Darier's disease. ${ }^{16}$ Our data show that in Darier's disease all aspects of cardiac contractile performance appear normal and in particular systolic function and contractility increase normally during exercise. These findings contrast with those of studies in mice in which one copy of the ATPA2A gene has been ablated, which do have impaired cardiac contractility. ${ }^{11}{ }^{12}$ Thus, the findings underscore the data obtained from the use of SERCA2a inhibitors that suggest wide interspecies variation in ability to compensate for sarcoplasmic reticulum dysfunction. ${ }^{25}$ Our data suggest that otherwise healthy human myocardium can compensate for a primary reduction in SERCA2 level or activity. Compensation probably occurs through decreased expression and increased phosphorylation of the inhibitory protein phospholamban and increased expression of the sarcolemmal $\mathrm{Na}^{+}-\mathrm{Ca}^{2+}$ exchanger. Upregulation of the normal allele or compensation by 


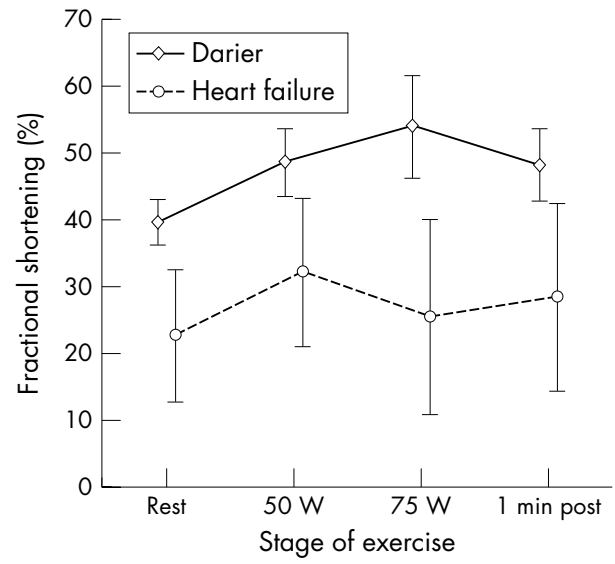

Figure 3 Left ventricular systolic function response at different stages of exercise in patients with Darier's disease and in patients with heart failure was determined by echocardiography at rest and in response to exercise with increasing workloads $(50 \mathrm{~W}, 75 \mathrm{~W})$ and one minute after cessation of exercise. Mean (SEM).

SERCAl or SERCA3 also conceivably plays a part. Mice heterozygous for a SERCA2 null allele, in which SERCA2 protein levels are reduced by about $35 \%$, do have a number of these adaptations, ${ }^{12}$ but presumably these are insufficient to completely rescue myocardial contractility because of the dependence on SERCA2 in the mouse. Similarly, discrepant phenotypes arising from mutations in phospholamban also highlight differences in the responses to perturbations in calcium handling in the heart between mouse and human. ${ }^{26}$

Our study has certain limitations inherent to clinical investigations. Firstly, we were unable to directly assess heart tissue in patients with Darier's disease, which would have allowed for direct measurement of SERCA2a activity and potential compensatory changes. Nevertheless, previous analyses of mutant SERCA2b isoforms are sufficient to predict a complete loss of function of one allele, such that the primary loss of cardiac SERCA2 activity in heterozygotes would be comparable with that described in heart failure. ${ }^{18} 19$ Secondly, we were restricted to non-invasive studies in these study subjects; we cannot exclude the presence of subtle abnormalities that may be detected by invasive tests of $\mathrm{dP} /$ $\mathrm{dT}_{\max }$ or direct measurement of the cellular function in Darier's disease.

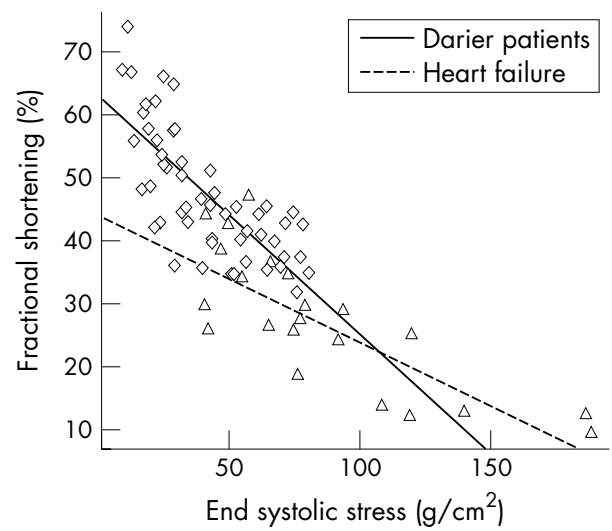

Figure 4 Contractility in patients with Darier's disease and patients with heart failure measured by the slope of the systolic shortening-end systolic stress relation. $\diamond$, Data points for patients with Darier's disease; $\Delta$, data points for patients with heart failure.
Although we could not directly examine the cellular and molecular effects of SERCA2 deficiency in the myocardium in the patients with Darier's disease, we believe that our observations have important implications for the role of SERCA2a in the aetiology and treatment of heart failure. The normal cardiac performance in human patients with Darier's disease suggests that the observed reduction in SERCA2a activity may not have a primary role in patients with heart failure. It is possible that studies in rodent models of treatments that seek to enhance SERCA2a activity in the treatment of heart failure may not be reliably extrapolated to human heart failure.

\section{ACKNOWLEDGEMENTS}

We thank the people who participated. BMM was a Nuffield Oxford Medical Fellow during the conduct of this study. This work was supported by grants from the Wellcome Trust and the British Heart Foundation.

\section{Authors' affiliations}

B M Mayosi ${ }^{*}$, A Kardos, C H Daviest, H Watkins, Department of Cardiovascular Medicine, University of Oxford, John Raddliffe Hospital, Oxford, UK

F Gumedze, Department of Statistical Sciences, University of Cape Town, Cape Town, South Africa

A Hovnanian, INSERM U563, Purpan Hospital, Toulouse, France S Burge, Department of Dermatology, The Churchill Hospital, Old Road, Headington, Oxford, UK

*Also the Cardiac Clinic, Department of Medicine, University of Cape Town, Cape Town, South Africa

†Also the Department of Cardiology, Oregon Health Science University, Portland, Oregon, USA

\section{REFERENCES}

1 Missiaen L, Wuytack F, Raeymaekers L, et al. Ca2+ extrusion across plasma membrane and $\mathrm{Ca} 2+$ uptake by intracellular stores. Pharmacol Ther 1991;50:191-232.

2 Frank KF, Bolck B, Erdmann E, et al. Sarcoplasmic reticulum Ca2+-ATPase modulates cardiac contraction and relaxation. Cardiovasc Res 2003;57:20-7

3 Arai $M$, Hirosuke $M$, Periasamy $M$. Sarcoplasmic reticulum gene expression in cardiac hypertrophy and heart failure. Circ Res 1994;74:555-64.

4 Hasenfuss G, Reinecke H, Studer R, et al. Relation between myocardial function and expression of sarcoplasmic reticulum calcium ATPase in failing and non-failing human myocardium. Circ Res 1994;75:434-42.

5 Pieske B, Kretschmann B, Meyer M, et al. Alterations in intracellular calcium handling associated with the inverse force-frequency relation in human dilated cardiomyopathy. Circulation 1995;92:1169-78.

6 Hasenfuss G. Alterations of calcium-regulatory proteins in heart failure. Cardiovasc Res 1998;37:279-89.

7 Davies $\mathrm{CH}$, Harding SE, Poole-Wilson PA. Cellular mechanisms of contractile dysfunction in human heart failure. Eur Heart J 1996;17:189-98.

8 Davies CH, Davia K, Bennett JG, et al. Reduced contraction and altered frequency response of isolated ventricular myocytes from patients with heart failure. Circulation 1995;92:2540-49.

9 Schmidt U, Haijar RJ, Helm PA, et al. Contribution of abnormal sarcoplasmic reticulum ATPase activity to systolic and diastolic dysfunction in human heart failure. J Mol Cell Cardiol 1998;30:1929-37.

10 Barry WH. Molecular inotropy: a future approach to the treatment of heart failure? Circulation 1999; 100:2303-4.

11 Periasamy M, Reed TD, Liu LH, et al. Impaired cardiac performance in heterozygous mice with a null mutation in the sarco(endo)plasmic reticulum Ca2+-ATPase isoform 2 (SERCA2) gene. J Biol Chem 1999;274:2556-62.

12 Ji Y, Lalli MJ, Babu GJ, et al. Disruption of a single copy of the SERCA2 gene results in altered $\mathrm{Ca}(2+)$ homeostasis and cardiomyocyte function. J Biol Chem 2000;275:38073-80.

13 Miyamoto MI, del Monte F, Schmidt U, et al. Adenoviral gene transfer of SERCA2a improves left-ventricular function in aortic-banded rats in transition to heart failure. Proc Natl Acad Sci USA 2000;97:793-8.

14 del Monte F, Harding SE, Schmidt U, et al. Restoration of contractile function in isolated cardiomyocytes from failing human hearts by gene transfer of SERCA2a. Circulation 1999;100:2308-11.

15 Schmidt AG, Haghighi K, Frank B, et al. Polymorphic SERCA2a variants do not account for inter-individual differences in phospholambanSERCA2a interactions in human heart failure. J Mol Cell Cardiol 2003;35:867-70

16 Tavadia S, Tait RC, McDonagh TA, et al. Platelet and cardiac function in Darier's disease. Clin Exp Dermatol 2001;26:696-9. 
17 Sakuntabhai A, Ruiz-Perez V, Carter S, et al. Mutations in ATP2A2, encoding a Ca2+ pump, cause Darier disease. Nat Genet 1999;21:271-7.

18 Ahn W, Lee MG, Kim KH, et al. Multiple effects of SERCA2b mutations associated with Darier's disease. J Biol Chem 2003;278:20795-801.

19 Dode L, Andersen JP, Leslie N, et al. Dissection of the functional differences between sarco(endo)plasmic reticulum Ca2+-ATPase (SERCA) 1 and 2 isoforms and characterization of Darier disease (SERCA2) mutants by steady-state and transient kinetic analyses. J Biol Chem 2003;278:47877-89.

20 Mayosi BM, Keavney B, Kardos A, et al. Electrocardiographic measures of left ventricular hypertrophy show greater heritability than echocardiographic left ventricular mass. Eur Heart J 2002;23:1963-71.

21 Armstrong WF. Stress echocardiography: introduction, history, and methods. Prog Cardiovasc Dis 1997;39:499-522.
22 Borow KM, Green LH, Grossman W, et al. Left ventricular end-systolic stressshortening and stress-length relations in human: normal values and sensitivity to inotropic state. Am J Cardiol 1982;50:1301-8.

23 Suman OE, Hasten D, Turner MJ, et al. Enhanced inotropic response to dobutamine in strength-trained subjects with left ventricular hypertrophy. J Appl Physiol 2000;88:534-9.

24 Sakuntabhai A, Burge S, Monk S, et al. Spectrum of novel ATP2A2 mutations in patients with Darier's disease. Hum Mol Genet 1999;8:1611-9.

25 Davia K, Davies CH, Harding SE. Effects of inhibition of sarcoplasmic reticulum calcium uptake on contraction in myocytes isolated from failing human ventricle. Cardiovasc Res 1997;33:88-97.

26 Haghighi K, Kolokathis F, Pater L, et al. Human phospholamban null results in lethal dilated cardiomyopathy revealing a critical difference between mouse and human. J Clin Invest 2003;111:869-76.

\section{IMAGES IN CARDIOLOGY}

\section{Massive pulmonary embolism without haemodynamic compromise caused by the presence of a} patent foramen ovale

A 36 year old man presented to hospital with a Fisher grade 2 subarachnoid haemorrhage (SAH). He was shown to have an $8.5 \times 12 \mathrm{~mm}$ right middle cerebral artery aneurysm which was clipped without complication.

Three days after craniotomy, he was alert with excellent gas exchange and was extubated. Three hours later he suddenly became cyanotic, profoundly hypoxic, and had a near respiratory arrest. He was immediately intubated and ventilated. He remained haemodynamically stable without the need for inotropic support; however, 100\% oxygen and nitric oxide $(20 \mathrm{ppm})$ was required to maintain the patient's $\mathrm{SaO}_{2}$ over $90 \%$. An urgent computed tomographic pulmonary angiogram (CTPA) showed the presence of a large "saddle embolus" extending into both left and right pulmonary arteries (panel A).

Thrombolysis was considered to be contraindicated because of his recent craniotomy, and as such he proceeded to have an open embolectomy via median sternotomy with cardiopulmonary bypass. Intraoperative transoesophageal echocardiogram revealed the presence of a patent foramen ovale (PFO) with torrential right to left shunt which accounted for his haemodynamic stability throughout. The presence of a PFO was also demonstrated on the CTPA when the images were reviewed postoperatively (panel B). During the 90 minute procedure a $25 \mathrm{~cm}$ intact embolus was removed from the main pulmonary trunk (panel C). Postembolectomy and PFO closure the gaseous exchange improved dramatically, and the patient proceeded to make a satisfactory recovery.

This case demonstrates that haemodynamic instability may not be present in massive pulmonary embolism if there is a large right to left intracardiac shunt. It also demonstrates that surgical embolectomy is a viable option when thrombolysis is contraindicated, and can be safely performed three days after craniotomy.

We would like to thank Mike Das Gupta for his photograph of the pulmonary embolus.
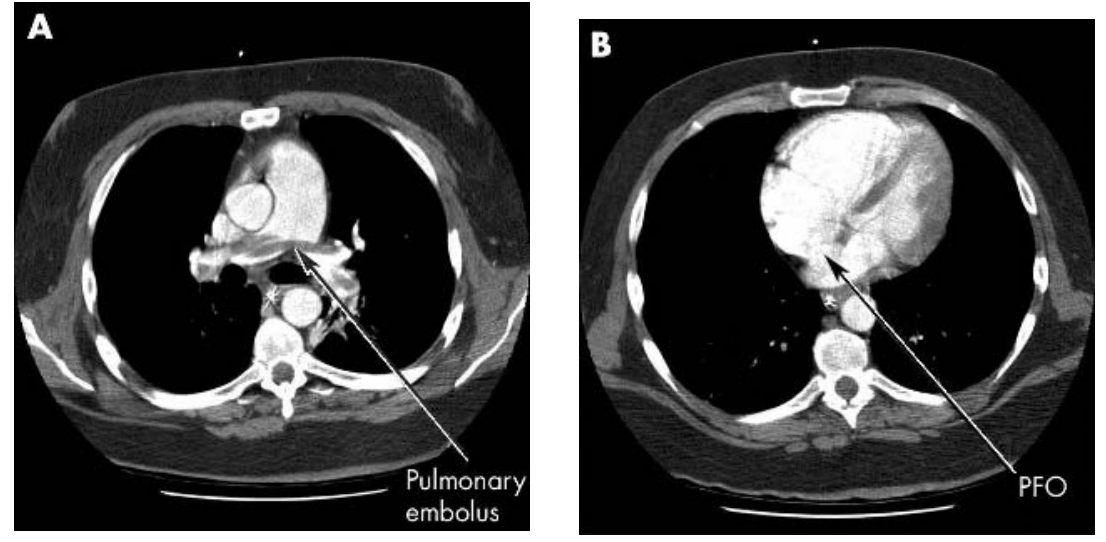

\section{C} PRECISION DYNAMICS CORP. SAN FERNANDO, CA 91340-3490 U.5.A. $<\{818\}$ 897. 1111

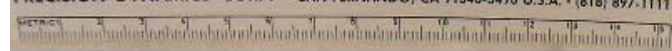

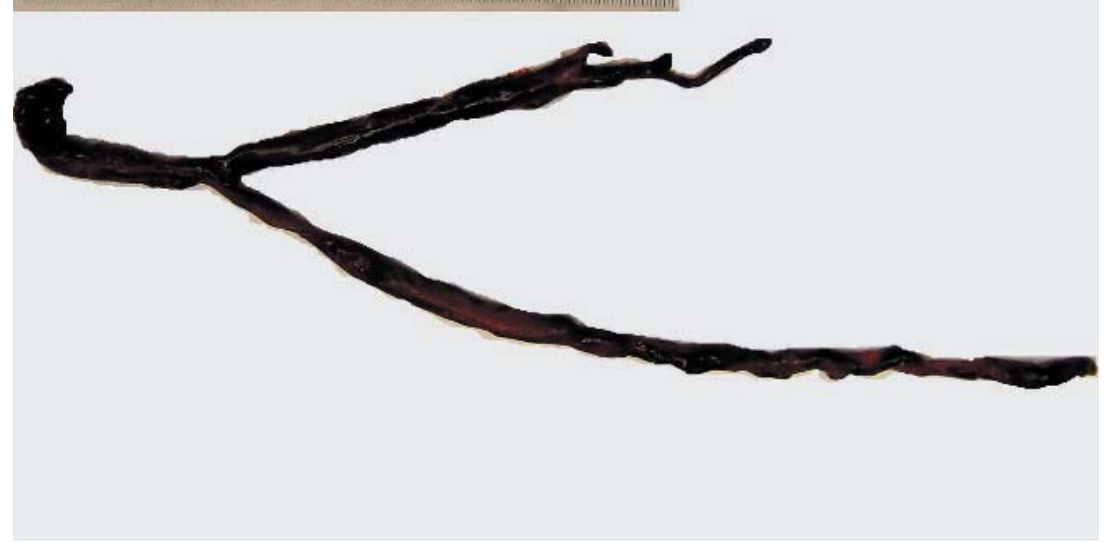

T R Keeble

$J$ Lewis

$\mathrm{K}$ M Ho

thomaskeeble@hotmail.com 